\title{
Positive Solutions for BVP of Fractional Differential Equation with Integral Boundary Conditions
}

\author{
Min Li, Jian-Ping Sun (D, and Ya-Hong Zhao \\ Department of Applied Mathematics, Lanzhou University of Technology, Lanzhou 730050, China \\ Correspondence should be addressed to Jian-Ping Sun; jpsun@lut.cn
}

Received 2 December 2019; Revised 6 March 2020; Accepted 30 March 2020; Published 23 April 2020

Academic Editor: Yuriy Rogovchenko

Copyright $\odot 2020$ Min Li et al. This is an open access article distributed under the Creative Commons Attribution License, which permits unrestricted use, distribution, and reproduction in any medium, provided the original work is properly cited.

In this paper, we consider a class of boundary value problems of nonlinear fractional differential equation with integral boundary conditions. By applying the monotone iterative method and some inequalities associated with Green's function, we obtain the existence of minimal and maximal positive solutions and establish two iterative sequences for approximating the solutions to the above problem. It is worth mentioning that these iterative sequences start off with zero function or linear function, which is useful and feasible for computational purpose. An example is also included to illustrate the main result of this paper.

\section{Introduction}

Fractional calculus has widespread applications in many fields of science and engineering, for example, viscoelasticity, continuum mechanics, bioengineering, rheology, electrical networks, control theory of dynamical systems, and optics and signal processing $[1,2]$.

In the past decades, the existence of solutions or positive solutions for boundary value problems (BVPs for short) of nonlinear fractional differential equations attracted considerable attention from many authors, see [3-19] and the references therein.

Recently, the monotone iterative method has been applied to study BVPs of nonlinear fractional differential equations. For example, in [20], Cui et al. discussed the BVP

$$
\begin{cases}\left(D_{0+}^{q} u\right)(t)+f(t, u(t))=0, & t \in(0,1), \\ u(0)=u^{\prime}(0)=0, & u(1)=0,\end{cases}
$$

where $2<q \leq 3$ and $D_{0+}^{q}$ denotes the standard Riemann-Liouville fractional derivative of order $q$. The authors obtained the existence of maximal and minimal solutions and the uniqueness result for BVP (1). In 2014, Sun and Zhao [21] investigated the following BVP with integral boundary conditions:

$$
\begin{cases}\left(D_{0}^{q} u\right)(t)+q(t) f(t, u(t))=0, & t \in(0,1), \\ u(0)=u^{\prime}(0)=0, & u(1)=\int_{0}^{1} g(s) u(s) \mathrm{d} s,\end{cases}
$$

where $2<q \leq 3$ and $D_{0+}^{q}$ is the standard Riemann-Liouville fractional derivative of order $q$. By means of the monotone iterative method, they proved the existence of a positive solution and established an iterative sequence for approximating the solution to BVP (2). For relevant results, one can refer to [22-25].

Motivated by the aforementioned works, in this paper, we consider the following BVP of nonlinear fractional differential equation with integral boundary conditions:

$$
\left\{\begin{array}{l}
\left({ }^{C} D_{0+}^{q} u\right)(t)+f(t, u(t))=0, t \in[0,1], \\
u^{\prime \prime}(0)=0, \\
\alpha u(0)-\beta u^{\prime}(0)=\int_{0}^{1} h_{1}(s) u(s) \mathrm{d} s, \\
\gamma u(1)+\delta\left({ }^{C} D_{0+}^{\sigma} u\right)(1)=\int_{0}^{1} h_{2}(s) u(s) \mathrm{d} s,
\end{array}\right.
$$

where ${ }^{C} D_{0+}^{q}$ and ${ }^{C} D_{0+}^{\sigma}$ denote the standard Caputo fractional derivatives of order $q$ and order $\sigma$, respectively. Throughout 
this paper, we always assume that $2<q \leq 3,0<\sigma \leq 1$ and $\alpha, \beta, \gamma$, and $\delta$ are nonnegative constants satisfying $0<\rho:=$ $(\alpha+\beta) \gamma+(\alpha \delta / \Gamma(2-\sigma))<\beta[\gamma+(\delta \Gamma(q) / \Gamma(q-\sigma))]$, and $f$ : $[0,1] \times[0,+\infty) \longrightarrow[0,+\infty)$ and $h_{i}(i=1,2):[0,1] \longrightarrow$ $[0,+\infty)$ are continuous.

The main tool used is the following theorem [26].

Theorem 1. Let $K$ be a normal cone of a Banach space $E$ and $v_{0} \preccurlyeq \omega_{0}$. Suppose that

$\left(a_{1}\right): T:\left[v_{0}, \omega_{0}\right] \longrightarrow E$ is completely continuous

$\left(a_{2}\right): T$ is monotone increasing on $\left[v_{0}, \omega_{0}\right]$

$\left(a_{3}\right): v_{0}$ is a lower solution of $T$, that is, $v_{0} \precsim T v_{0}$

$\left(a_{4}\right): \omega_{0}$ is an upper solution of $T$, that is, $T \omega_{0} \precsim \omega_{0}$

Then, the iterative sequences

$$
\begin{aligned}
v_{n} & =T v_{n-1}, \\
\omega_{n} & =T \omega_{n-1}, \quad n=1,2,3, \ldots,
\end{aligned}
$$

satisfy

$$
v_{0} \precsim v_{1} \precsim \cdots \precsim v_{n} \preccurlyeq \cdots \precsim \omega_{n} \preccurlyeq \cdots \precsim \omega_{1} \preccurlyeq \omega_{0},
$$

and converge to, respectively, $v$ and $\omega \in\left[v_{0}, \omega_{0}\right]$, which are fixed points of $T$.

\section{Preliminaries}

First, we present the definitions of Riemann-Liouville fractional integral and fractional derivative and Caputo fractional derivative on a finite interval of the real line, which may be found in [1].

In this section, we always assume that $\mathbb{N}=\{1,2,3, \ldots\}, \mu, \nu>0$ and $[\mu]$ denotes the integer part of $\mu$.

Definition 1. The Riemann-Liouville fractional integral $I_{0+}^{\mu} u$ of order $\mu$ on $[0,1]$ is defined by

$$
\left(I_{0+}^{\mu} u\right)(t):=\frac{1}{\Gamma(\mu)} \int_{0}^{t} \frac{u(s) \mathrm{d} s}{(t-s)^{1-\mu}} .
$$

Definition 2. The Riemann-Liouville fractional derivative $D_{0+}^{\mu} u$ of order $\mu$ on $[0,1]$ is defined by

$$
\begin{aligned}
\left(D_{0+}^{\mu} u\right)(t) & :=\left(\frac{\mathrm{d}}{\mathrm{d} t}\right)^{n}\left(I_{0+}^{n-\mu} u\right)(t) \\
& =\frac{1}{\Gamma(n-\mu)}\left(\frac{\mathrm{d}}{\mathrm{d} t}\right)^{n} \int_{0}^{t} \frac{u(s) \mathrm{d} s}{(t-s)^{(\mu-n+1)}},
\end{aligned}
$$

where $n=[\mu]+1$.

Definition 3. Let $D_{0+}^{\mu}[u(s)](t) \equiv\left(D_{0+}^{\mu} u\right)(t)$ be the Riemann-Liouville fractional derivative of order $\mu$. Then, the
Caputo fractional derivative ${ }^{C} D_{0+}^{\mu} u$ of order $\mu$ on $[0,1]$ is defined by

$$
\left({ }^{C} D_{0+}^{\mu} u\right)(t):=\left(D_{0+}^{\mu}\left[u(s)-\sum_{k=0}^{n-1} \frac{u^{(k)}(0)}{k !} s^{k}\right]\right)(t),
$$

where

$$
n=\left\{\begin{array}{l}
{[\mu]+1, \quad \mu \notin \mathbb{N},} \\
\mu, \mu \in \mathbb{N} .
\end{array}\right.
$$

Lemma 1 (see [2]). Let $v>\mu$. Then, the equation $\left({ }^{C} D_{0+}^{\mu} I_{0+}^{v} u\right)(t)=\left(I_{0+}^{\nu-\mu} u\right)(t), t \in[0,1] \quad$ is satisfied for $u \in C[0,1]$.

Lemma 2 (see [1]). Let $n$ be given by (9). Then, the following relations hold:

(1) For $k \in\{0,1,2, \ldots, n-1\},{ }^{C} D_{0+}^{\mu} t^{k}=0$.

(2) If $\nu>n$, then ${ }^{C} D_{0+}^{\mu} t^{\nu-1}=(\Gamma(\nu) / \Gamma(\nu-\mu)) t^{\nu-\mu-1}$.

For convenience, we denote

$P_{i}=\frac{1}{\rho} \int_{0}^{1}(\alpha s+\beta) h_{i}(s) \mathrm{d} s$,

$Q_{i}=\frac{1}{\rho \Gamma(2-\sigma)} \int_{0}^{1}[\gamma \Gamma(2-\sigma)(1-s)+\delta] h_{i}(s) \mathrm{d} s, \quad i=1,2$.

Lemma 3. Let $\left(1-Q_{1}\right)\left(1-P_{2}\right) \neq P_{1} Q_{2}$. Then, for any $y \in C[0,1]$, the $B V P$

$$
\left\{\begin{array}{l}
\left({ }^{C} D_{0+}^{q} u\right)(t)+y(t)=0, t \in[0,1] \\
u^{\prime \prime}(0)=0 \\
\alpha u(0)-\beta u^{\prime}(0)=\int_{0}^{1} h_{1}(s) u(s) \mathrm{d} s \\
\gamma u(1)+\delta\left({ }^{C} D_{0+}^{\sigma} u\right)(1)=\int_{0}^{1} h_{2}(s) u(s) \mathrm{d} s
\end{array}\right.
$$

has a unique solution

$$
u(t)=\int_{0}^{1} H(t, s) y(s) \mathrm{d} s, \quad t \in[0,1] .
$$

Here,

$$
\begin{array}{r}
H(t, s)=G(t, s)+\sum_{i=1}^{2} \phi_{i}(t) \int_{0}^{1} G(\tau, s) h_{i}(\tau) \mathrm{d} \tau, \\
(t, s) \in[0,1] \times[0,1],
\end{array}
$$

where 


$$
\begin{gathered}
G(t, s)=\frac{\alpha t+\beta}{\rho}\left[\frac{\gamma(1-s)^{q-1}}{\Gamma(q)}+\frac{\delta(1-s)^{q-\sigma-1}}{\Gamma(q-\sigma)}\right]- \begin{cases}\frac{(t-s)^{q-1}}{\Gamma(q)}, & 0 \leq s \leq t \leq 1, \\
0, & 0 \leq t \leq s \leq 1,\end{cases} \\
\phi_{1}(t)=\frac{\Gamma(2-\sigma) Q_{2}(\alpha t+\beta)+\left(1-P_{2}\right)[\gamma \Gamma(2-\sigma)(1-t)+\delta]}{\rho \Gamma(2-\sigma)\left[\left(1-Q_{1}\right)\left(1-P_{2}\right)-P_{1} Q_{2}\right]}, \quad t \in[0,1], \\
\phi_{2}(t)=\frac{\Gamma(2-\sigma)\left(1-Q_{1}\right)(\alpha t+\beta)+P_{1}[\gamma \Gamma(2-\sigma)(1-t)+\delta]}{\rho \Gamma(2-\sigma)\left[\left(1-Q_{1}\right)\left(1-P_{2}\right)-P_{1} Q_{2}\right]}, \quad t \in[0,1] .
\end{gathered}
$$

Proof. In view of the equation in (11), Theorem 3.24 [1], and $u^{\prime \prime}(0)=0$, we have

$$
u(t)=-\left(I_{0+}^{q} y\right)(t)+u(0)+u^{\prime}(0) t, \quad t \in[0,1] .
$$

By (15), Lemma 1, and Lemma 2, we obtain

$$
\left({ }^{C} D_{0+}^{\sigma} u\right)(t)=-\left(I_{0+}^{q-\sigma} y\right)(t)+\frac{u^{\prime}(0)}{\Gamma(2-\sigma)} t^{1-\sigma}, \quad t \in[0,1] .
$$

It follows from (15) and (16) and the boundary conditions in (11) that

$$
\begin{aligned}
u(0) & =\frac{1}{\rho}\left[\beta \gamma\left(I_{0+}^{q} y\right)(1)+\beta \delta\left(I_{0+}^{q-\sigma} y\right)(1)+\frac{\gamma \Gamma(2-\sigma)+\delta}{\Gamma(2-\sigma)} \int_{0}^{1} h_{1}(s) u(s) \mathrm{d} s+\beta \int_{0}^{1} h_{2}(s) u(s) \mathrm{d} s\right], \\
u^{\prime}(0) & =\frac{1}{\rho}\left[\alpha \gamma\left(I_{0+}^{q} y\right)(1)+\alpha \delta\left(I_{0+}^{q-\sigma} y\right)(1)-\gamma \int_{0}^{1} h_{1}(s) u(s) \mathrm{d} s+\alpha \int_{0}^{1} h_{2}(s) u(s) \mathrm{d} s\right],
\end{aligned}
$$

which together with (15) shows that

$$
\begin{aligned}
u(t)= & \int_{0}^{t}\left\{\frac{\alpha t+\beta}{\rho}\left[\frac{\gamma(1-s)^{q-1}}{\Gamma(q)}+\frac{\delta(1-s)^{q-\sigma-1}}{\Gamma(q-\sigma)}\right]-\frac{(t-s)^{q-1}}{\Gamma(q)}\right\} y(s) \mathrm{d} s \\
& +\int_{t}^{1}\left\{\frac{\alpha t+\beta}{\rho}\left[\frac{\gamma(1-s)^{q-1}}{\Gamma(q)}+\frac{\delta(1-s)^{q-\sigma-1}}{\Gamma(q-\sigma)}\right]\right\} y(s) \mathrm{d} s \\
& +\frac{\gamma \Gamma(2-\sigma)(1-t)+\delta}{\rho \Gamma(2-\sigma)} \int_{0}^{1} h_{1}(s) u(s) \mathrm{d} s+\frac{\alpha t+\beta}{\rho} \int_{0}^{1} h_{2}(s) u(s) \mathrm{d} s \\
= & \int_{0}^{1} G(t, s) y(s) \mathrm{d} s+\frac{\gamma \Gamma(2-\sigma)(1-t)+\delta}{\rho \Gamma(2-\sigma)} \int_{0}^{1} h_{1}(s) u(s) \mathrm{d} s \\
& +\frac{\alpha t+\beta}{\rho} \int_{0}^{1} h_{2}(s) u(s) \mathrm{d} s, \quad t \in[0,1] .
\end{aligned}
$$

From (18), we get

$$
\begin{array}{r}
\left(1-Q_{1}\right) \int_{0}^{1} h_{1}(s) u(s) \mathrm{d} s-P_{1} \int_{0}^{1} h_{2}(s) u(s) \mathrm{d} s=\int_{0}^{1} h_{1}(s) \int_{0}^{1} G(s, \tau) y(\tau) \mathrm{d} \tau \mathrm{d} s, \\
-Q_{2} \int_{0}^{1} h_{1}(s) u(s) \mathrm{d} s+\left(1-P_{2}\right) \int_{0}^{1} h_{2}(s) u(s) \mathrm{d} s=\int_{0}^{1} h_{2}(s) \int_{0}^{1} G(s, \tau) y(\tau) \mathrm{d} \tau \mathrm{d} s,
\end{array}
$$

and so, 


$$
\begin{aligned}
& \int_{0}^{1} h_{1}(s) u(s) \mathrm{d} s=\frac{\left(1-P_{2}\right) \int_{0}^{1} h_{1}(s) \int_{0}^{1} G(s, \tau) y(\tau) \mathrm{d} \tau \mathrm{d} s+P_{1} \int_{0}^{1} h_{2}(s) \int_{0}^{1} G(s, \tau) y(\tau) \mathrm{d} \tau \mathrm{d} s}{\left(1-Q_{1}\right)\left(1-P_{2}\right)-\left(P_{1} Q_{2}\right)}, \\
& \int_{0}^{1} h_{2}(s) u(s) \mathrm{d} s=\frac{Q_{2} \int_{0}^{1} h_{1}(s) \int_{0}^{1} G(s, \tau) y(\tau) \mathrm{d} \tau \mathrm{d} s+\left(1-Q_{1}\right) \int_{0}^{1} h_{2}(s) \int_{0}^{1} G(s, \tau) y(\tau) \mathrm{d} \tau \mathrm{d} s}{\left(1-Q_{1}\right)\left(1-P_{2}\right)-\left(P_{1} Q_{2}\right)},
\end{aligned}
$$

which together with (18) implies that

$$
\begin{aligned}
u(t) & =\int_{0}^{1} G(t, s) y(s) \mathrm{d} s+\sum_{i=1}^{2} \phi_{i}(t) \int_{0}^{1} h_{i}(s) \int_{0}^{1} G(s, \tau) y(\tau) \mathrm{d} \tau \mathrm{d} s \\
& =\int_{0}^{1} G(t, s) y(s) \mathrm{d} s+\sum_{i=1}^{2} \phi_{i}(t) \int_{0}^{1} h_{i}(\tau) \int_{0}^{1} G(\tau, s) y(s) \mathrm{d} s \mathrm{~d} \tau \\
& =\int_{0}^{1} G(t, s) y(s) \mathrm{d} s+\sum_{i=1}^{2} \phi_{i}(t) \int_{0}^{1} y(s) \int_{0}^{1} G(\tau, s) h_{i}(\tau) \mathrm{d} \tau \mathrm{d} s \\
& =\int_{0}^{1}\left[G(t, s)+\sum_{i=1}^{2} \phi_{i}(t) \int_{0}^{1} G(\tau, s) h_{i}(\tau) \mathrm{d} \tau\right] y(s) \mathrm{d} s \\
& =\int_{0}^{1} H(t, s) y(s) \mathrm{d} s, \quad t \in[0,1] .
\end{aligned}
$$

In what follows, we let

$$
\begin{aligned}
& g(s)=\frac{\alpha+\beta}{\rho}\left[\frac{\gamma(1-s)^{q-1}}{\Gamma(q)}+\frac{\delta(1-s)^{q-\sigma-1}}{\Gamma(q-\sigma)}\right], \quad s \in[0,1], \\
& \eta(s)=\frac{\beta \delta \Gamma(q)-\Gamma(q-\sigma)(\rho-\beta \gamma)}{(\alpha+\beta)[\gamma \Gamma(q-\sigma)+\delta \Gamma(q)]}(1-s)^{\sigma}, \quad s \in[0,1] .
\end{aligned}
$$

Lemma 4. $G(t, s)$ satisfies the following properties:

(1) $G(t, s) \leq g(s),(t, s) \in[0,1] \times[0,1]$.

(2) $G(t, s) \geq \eta(s) g(s),(t, s) \in[0,1] \times[0,1]$.

Proof. Since (1) is obvious, we only need to prove that (2) holds.

First, it is clear that $G(t, 1) \geq \eta(1) g(1)$ for $t \in[0,1]$.

Now, we verify that $G(t, s) \geq \eta(s) g(s)$ for $(t, s) \in[0,1] \times[0,1)$. In fact, if $s \leq t$, then

$$
\begin{aligned}
\frac{G(t, s)}{g(s)} & =\frac{(\alpha t+\beta)\left[\gamma \Gamma(q-\sigma)(1-s)^{q-1}+\delta \Gamma(q)(1-s)^{q-\sigma-1}\right]-\rho \Gamma(q-\sigma)(t-s)^{q-1}}{(\alpha+\beta)\left[\gamma \Gamma(q-\sigma)(1-s)^{q-1}+\delta \Gamma(q)(1-s)^{q-\sigma-1}\right]} \\
& \geq \frac{\beta \gamma \Gamma(q-\sigma)(1-s)^{\sigma}+\beta \delta \Gamma(q)-\rho \Gamma(q-\sigma)(1-s)^{\sigma}}{(\alpha+\beta)\left[\gamma \Gamma(q-\sigma)(1-s)^{\sigma}+\delta \Gamma(q)\right]} \\
& \geq \frac{\beta \delta \Gamma(q)-\Gamma(q-\sigma)(\rho-\beta \gamma)}{(\alpha+\beta)[\gamma \Gamma(q-\sigma)+\delta \Gamma(q)]}(1-s)^{\sigma} \\
& =\eta(s),
\end{aligned}
$$

and if $t \leq s$, then

$$
\begin{aligned}
\frac{G(t, s)}{g(s)} & =\frac{(\alpha t+\beta)\left[\gamma \Gamma(q-\sigma)(1-s)^{q-1}+\delta \Gamma(q)(1-s)^{q-\sigma-1}\right]}{(\alpha+\beta)\left[\gamma \Gamma(q-\sigma)(1-s)^{q-1}+\delta \Gamma(q)(1-s)^{q-\sigma-1}\right]} \\
& \geq \frac{\beta \gamma \Gamma(q-\sigma)(1-s)^{\sigma}+\beta \delta \Gamma(q)}{(\alpha+\beta)\left[\gamma \Gamma(q-\sigma)(1-s)^{\sigma}+\delta \Gamma(q)\right]} \\
& \geq \frac{\beta \gamma \Gamma(q-\sigma)(1-s)^{\sigma}+\beta \delta \Gamma(q)-\rho \Gamma(q-\sigma)(1-s)^{\sigma}}{(\alpha+\beta)\left[\gamma \Gamma(q-\sigma)(1-s)^{\sigma}+\delta \Gamma(q)\right]} \\
& \geq \frac{\beta \delta \Gamma(q)-\Gamma(q-\sigma)(\rho-\beta \gamma)}{(\alpha+\beta)[\gamma \Gamma(q-\sigma)+\delta \Gamma(q)]}(1-s)^{\sigma} \\
& =\eta(s) .
\end{aligned}
$$


By the definition of $\eta$ and the condition $0<\rho<\beta[\gamma+(\delta \Gamma(q) / \Gamma(q-\sigma))]$, we may obtain the following remark.

Remark 1. $\eta(s) \geq 0$ and $\eta(s) \neq \equiv$ for $s \in[0,1]$.

In the remainder of this paper, we always assume that the following condition is fulfilled:

$$
(C 1) Q_{1}<1, \quad P_{2}<1,\left(1-Q_{1}\right)\left(1-P_{2}\right)>P_{1} Q_{2} .
$$

Now, we define

$$
x(t)=1+\sum_{i=1}^{2} \int_{0}^{1} h_{i}(\tau) \mathrm{d} \tau \phi_{i}(t), t \in[0,1] .
$$

Lemma 5. $H(t, s)$ has the following property:

$$
x(t) \eta(s) g(s) \leq H(t, s) \leq x(t) g(s),(t, s) \in[0,1] \times[0,1] .
$$

Proof. In view of the definition of $H(t, s)$ and Lemma 4 , it is obvious.

\section{Main Results}

For convenience, we let

$$
\begin{aligned}
& A=\frac{1}{\int_{0}^{1} g(s) \mathrm{d} s}, \\
& a=\max _{t \in[0,1]} x(t) .
\end{aligned}
$$

Theorem 2. Assume that $f(t, 0) \equiv 0$ for $t \in[0,1]$ and the following condition is satisfied:

$$
\text { (C2) } f\left(t, u_{1}\right) \leq f\left(t, u_{2}\right) \leq A, \quad 0 \leq t \leq 1,0 \leq u_{1} \leq u_{2} \leq a .
$$

Then, $B V P(3)$ possesses a minimal positive solution $v$ and a maximal positive solution $\omega$.

Proof. Let $E=C[0,1]$ be equipped with the norm

$$
\begin{aligned}
\|u\| & =\max _{t \in[0,1]}|u(t)|, \\
K & =\{u \in E: u(t) \geq 0, t \in[0,1]\} .
\end{aligned}
$$

Then, $K$ is a normal cone in Banach space $E$. Note that this induces an order relation " $\lesssim$ " in $E$ by defining $u \lesssim v$ if and only if $v-u \in K$.

Now, we define $v_{0}(t) \equiv 0$ and $\omega_{0}(t)=x(t)$ for $t \in[0,1]$ and $T:\left[v_{0}, \omega_{0}\right] \longrightarrow K$ by

$$
(T u)(t)=\int_{0}^{1} H(t, s) f(s, u(s)) \mathrm{d} s, \quad u \in\left[v_{0}, \omega_{0}\right], t \in[0,1] .
$$

Then, it is easy to know that $T:\left[v_{0}, \omega_{0}\right] \longrightarrow K$ is completely continuous, and fixed points of $T$ are nonnegative solutions of BVP (3).
Step 1. We assert that $T$ is monotone increasing on $\left[v_{0}, \omega_{0}\right]$. Let $u, v \in\left[v_{0}, \omega_{0}\right]$ and $u \lesssim v$. Then, $0 \leq u(t) \leq v(t) \leq a$ for $t \in[0,1]$, which together with $(C 2)$ implies that

$$
\begin{aligned}
(T u)(t) & =\int_{0}^{1} H(t, s) f(s, u(s)) \mathrm{d} s \\
& \leq \int_{0}^{1} H(t, s) f(s, v(s)) \mathrm{d} s \\
& =(T v)(t), \quad t \in[0,1] .
\end{aligned}
$$

This shows that $T u \lesssim T v$.

Step 2. We prove that $v_{0}$ is a lower solution of $T$.

For any $t \in[0,1]$, we have

$$
\left(T v_{0}\right)(t)=\int_{0}^{1} H(t, s) f(s, 0) \mathrm{d} s \geq 0=v_{0}(t),
$$

which indicates that $v_{0} \lesssim T v_{0}$.

Step 3. We show that $\omega_{0}$ is an upper solution of $T$.

In view of Lemma 5 and (C2), we get

$$
\begin{aligned}
\left(T \omega_{0}\right)(t) & =\int_{0}^{1} H(t, s) f\left(s, \omega_{0}(s)\right) \mathrm{d} s \\
& \leq A x(t) \int_{0}^{1} g(s) \mathrm{d} s \\
& =\omega_{0}(t), \quad t \in[0,1],
\end{aligned}
$$

which implies that $T \omega_{0} \precsim \omega_{0}$.

Step 4. We claim that BVP (3) possesses a minimal positive solution $v$ and a maximal positive solution $\omega$.

In fact, if we construct sequences $\left\{v_{n}\right\}_{n=0}^{\infty}$ and $\left\{\omega_{n}\right\}_{n=0}^{\infty}$ as in the following,

$$
\begin{aligned}
v_{n} & =T v_{n-1}, \\
\omega_{n} & =T \omega_{n-1}, \quad n=1,2,3, \ldots,
\end{aligned}
$$

then it follows from Theorem 1 that

$$
v_{0} \preccurlyeq v_{1} \preccurlyeq \cdots \precsim v_{n} \preccurlyeq \cdots \precsim \omega_{n} \preccurlyeq \cdots \precsim \omega_{1} \precsim \omega_{0},
$$

and $\left\{v_{n}\right\}_{n=0}^{\infty}$ and $\left\{\omega_{n}\right\}_{n=0}^{\infty}$ converge to, respectively, $v$ and $\omega \in\left[v_{0}, \omega_{0}\right]$, which are nonnegative solutions of BVP (3).

In view of Lemma 5, Remark 1, the definitions of $x(t)$ and $g(s)$, and the assumption $f(t, 0) \equiv 0$ for $t \in[0,1]$, we get

$$
\begin{aligned}
\left(T v_{0}\right)(t) & =\int_{0}^{1} H(t, s) f(s, 0) \mathrm{d} s \\
& \geq x(t) \int_{0}^{1} \eta(s) g(s) f(s, 0) \mathrm{d} s \\
& >0, \quad t \in[0,1],
\end{aligned}
$$

and so,

$$
0<\left(T v_{0}\right)(t) \leq(T v)(t)=v(t) \leq \omega(t), \quad t \in[0,1],
$$

which shows that $v$ and $\omega$ are positive solutions of the BVP (3). 
Moreover, if $u \in\left[v_{0}, \omega_{0}\right]$ is a positive solution of the BVP (3), then it follows from the fact $T$ is monotone increasing on $\left[v_{0}, \omega_{0}\right]$ that $v \lesssim u \lesssim \omega$. This indicates that $v$ and $\omega$ are minimal and maximal positive solutions of the BVP (3), respectively.

Example 1. Consider the following BVP:

$$
\left\{\begin{array}{l}
\left({ }^{C} D_{0+}^{(5 / 2)} u\right)(t)+\frac{\sqrt{\pi}}{3}\left[u^{2}(t) \frac{14741}{13456} \sin (\pi t)\right]=0, \quad t \in[0,1], \\
u^{\prime \prime}(0)=0, \\
u(0)-3 u^{\prime}(0)=\int_{0}^{1} s u(s) \mathrm{d} s, \\
2 u(1)+u^{\prime}(1)=\frac{1}{2} \int_{0}^{1} s u(s) \mathrm{d} s .
\end{array}\right.
$$

Since $q=(5 / 2), \sigma=\alpha=\delta=1, \beta=3$, and $\gamma=2$, a simple calculation shows that

$$
\begin{aligned}
0<\rho & =(\alpha+\beta) \gamma+\frac{\alpha \delta}{\Gamma(2-\sigma)}=9<\beta\left[\gamma+\frac{\delta \Gamma(q)}{\Gamma(q-\sigma)}\right] \\
& =\frac{21}{2}, A=\frac{15 \sqrt{\pi}}{16} .
\end{aligned}
$$

Moreover, in view of $h_{1}(s)=s$ and $h_{2}(s)=s / 2$ for $s \in[0,1]$, we get

$$
\begin{aligned}
& P_{1}=\frac{11}{54}, \\
& P_{2}=\frac{11}{108}, \\
& Q_{1}=\frac{5}{54}, \\
& Q_{2}=\frac{5}{108},
\end{aligned}
$$

which indicates that $(C 1)$ is fulfilled. At the same time, we also obtain that

$$
a=\max _{t \in[0,1]} x(t)=\frac{38}{29} .
$$

Now, if we let $f(t, u)=(\sqrt{\pi} / 3)\left[u^{2}+\right.$ $(14741 / 13456) \sin (\pi t)],(t, u) \in[0,1] \times[0,+\infty)$, then it is easy to know that $f:[0,1] \times[0,+\infty) \longrightarrow[0,+\infty)$ is continuous, $f(t, 0) \not \equiv 0$ for $t \in[0,1]$, and $(C 2)$ is fulfilled. Therefore, it follows from Theorem 2 that BVP (39) possesses a minimal positive solution $v$ and a maximal positive solution $\omega$. In addition, the two iterative schemes are as follows:

$$
\begin{aligned}
v_{0}(t) \equiv & 0, t \in[0,1] \\
v_{n+1}(t)= & \int_{0}^{1}\left\{\frac{32 t-96}{3045}\left[(-s)^{(7 / 2)}+(t-s)^{(5 / 2)} s\right]+\frac{16 t^{2}-48 t}{609}(t-s)^{(5 / 2)}+\frac{48 t+320}{783}(1-s)^{(3 / 2)}+\frac{12 t+80}{261}(1-s)^{(1 / 2)}\right\}\left[v_{n}^{2}(s)\right. \\
& \left.+\frac{14741}{13456} \sin (\pi s)\right] \mathrm{d} s-\frac{4}{9} \int_{0}^{t}(t-s)^{(3 / 2)}\left[v_{n}^{2}(s)+\frac{14741}{13456} \sin (\pi s)\right] \mathrm{d} s, \quad t \in[0,1], n=0,1,2, \ldots ; \\
\omega_{0}(t)= & \frac{-3 t+38}{29}, \quad t \in[0,1], \\
\omega_{n+1}(t)= & \int_{0}^{1}\left\{\frac{32 t-96}{3045}\left[(-s)^{(7 / 2)}+(t-s)^{(5 / 2)} s\right]+\frac{16 t^{2}-48 t}{609}(t-s)^{(5 / 2)}+\frac{48 t+320}{783}(1-s)^{(3 / 2)}+\frac{12 t+80}{261}(1-s)^{(1 / 2)}\right\}\left[\omega_{n}^{2}(s)\right. \\
& \left.+\frac{14741}{13456} \sin (\pi s)\right] \mathrm{d} s-\frac{4}{9} \int_{0}^{t}(t-s)^{(3 / 2)}\left[\omega_{n}^{2}(s)+\frac{14741}{13456} \sin (\pi s)\right] \mathrm{d} s, \quad t \in[0,1], n=0,1,2, \ldots
\end{aligned}
$$




\section{Data Availability}

No data were used to support this study.

\section{Conflicts of Interest}

The authors declare that there are no conflicts of interest regarding the publication of this paper.

\section{Acknowledgments}

This work was supported by the National Natural Science Foundation of China (Grant 11661049).

\section{References}

[1] A. A. Kilbas, H. M. Srivastava, and J. J. Trujillo, "Theory and applications of fractional differential equations," vol. 204 of North-Holland Mathematics Studies, Elsevier Science, Amsterdam, The Netherlands, 2006.

[2] S. G. Samko, A. A. Kilbas, and O. I. Marichev, Fractional Integrals and Derivatives: Theory and Applications, Gordon and Breach Science, Yverdon, Switzerland, 1993.

[3] R. P. Agarwal, B. Ahmad, D. A. Garout, and A. Alsaedi, "Existence results for coupled nonlinear fractional differential equations equipped with nonlocal coupled flux and multipoint boundary conditions," Chaos, Solitons \& Fractals, vol. 102, pp. 149-161, 2017.

[4] R. P. Agarwal, M. Benchohra, and S. Hamani, "Boundary value problems for fractional differential equations," Georgian Mathematical Journal, vol. 16, no. 3, pp. 401-411, 2009.

[5] Z. Bai and T. Qiu, "Existence of positive solution for singular fractional differential equation," Applied Mathematics and Computation, vol. 215, no. 7, pp. 2761-2767, 2009.

[6] A. Cabada, S. Aleksić, T. V. Tomović, and S. Dimitrijević, "Existence of solutions of nonlinear and non-local fractional boundary value problems," Mediterranean Journal of Mathematics, vol. 16, no. 119, pp. 1-18, 2019.

[7] A. Cabada and G. Wang, "Positive solutions of nonlinear fractional differential equations with integral boundary value conditions," Journal of Mathematical Analysis and Applications, vol. 389, no. 1, pp. 403-411, 2012.

[8] A. Guezane-Lakoud and S. Bensebaa, "Solvability of a fractional boundary value problem with fractional derivative condition," Arabian Journal of Mathematics, vol. 3, no. 1, pp. 39-48, 2014.

[9] A. Guezane-Lakoud and R. Khaldi, "Existence results for a fractional boundary value problem with fractional Lidstone conditions," Journal of Applied Mathematics and Computing, vol. 49, no. 1-2, pp. 261-268, 2015.

[10] A. Guezane-Lakoud and R. Khaldi, "Solvability of a fractional boundary value problem with fractional integral condition," Nonlinear Analysis: Theory, Methods \& Applications, vol. 75, no. 4, pp. 2692-2700, 2012.

[11] D. Jiang and C. Yuan, "The positive properties of the green function for dirichlet-type boundary value problems of nonlinear fractional differential equations and its application," Nonlinear Analysis: Theory, Methods \& Applications, vol. 72, no. 2, pp. 710-719, 2010.

[12] C. F. Li, X. N. Luo, and Y. Zhou, "Existence of positive solutions of the boundary value problem for nonlinear fractional differential equations," Computers \& Mathematics with Applications, vol. 59, no. 3, pp. 1363-1375, 2010.
[13] C.-M. Su, J.-P. Sun, and Y.-H. Zhao, "Existence and uniqueness of solutions for BVP of nonlinear fractional differential equation," International Journal of Differential Equations, vol. 2017, Article ID 4683581, 7 pages, 2017.

[14] J. Xu, Z. Wei, and W. Dong, "Uniqueness of positive solutions for a class of fractional boundary value problems," Applied Mathematics Letters, vol. 25, no. 3, pp. 590-593, 2012.

[15] W. Yang, "Positive solutions for nonlinear Caputo fractional differential equations with integral boundary conditions," Journal of Applied Mathematics and Computing, vol. 44, no. 12, pp. 39-59, 2014.

[16] J.-R. Yue, J.-P. Sun, and S. Zhang, "Existence of positive solution for BVP of nonlinear fractional differential equation," Discrete Dynamics in Nature and Society, vol. 2015, Article ID 736108, 6 pages, 2015.

[17] S. Zhang, "Positive solutions for boundary-value problems of nonlinear fractional differential equations," Electronic Journal of Differential Equations, vol. 2006, no. 36, pp. 1-12, 2006.

[18] X. Zhang, L. Wang, and Q. Sun, "Existence of positive solutions for a class of nonlinear fractional differential equations with integral boundary conditions and a parameter," Applied Mathematics and Computation, vol. 226, no. 1-2, pp. 708-718, 2014.

[19] K. Zhao and P. Gong, "Existence of positive solutions for a class of higher-order Caputo fractional differential equation," Qualitative Theory of Dynamical Systems, vol. 14, no. 1, pp. 157-171, 2015.

[20] Y. Cui, Q. Sun, and X. Su, "Monotone iterative technique for nonlinear boundary value problems of fractional order $p \in(2,3], "$ Advances in Difference Equations, vol. 2017, no. 248, pp. 1-12, 2017.

[21] Y. Sun and M. Zhao, "Positive solutions for a class of fractional differential equations with integral boundary conditions," Applied Mathematics Letters, vol. 34, pp. 17-21, 2014.

[22] C. Hu, B. Liu, and S. Xie, "Monotone iterative solutions for nonlinear boundary value problems of fractional differential equation with deviating arguments," Applied Mathematics and Computation, vol. 222, pp. 72-81, 2013.

[23] M. Jiang and S. Zhong, "Successively iterative method for fractional differential equations with integral boundary conditions," Applied Mathematics Letters, vol. 38, pp. 94-99, 2014.

[24] F. A. Mcrae, "Monotone method for periodic boundary value problems of Caputo fractional differential equations," Communications in Applied Analysis, vol. 14, no. 1, pp. 73-80, 2010.

[25] X. Zhang, L. Liu, Y. Wu, and Y. Lu, "The iterative solutions of nonlinear fractional differential equations," Applied Mathematics and Computation, vol. 219, no. 9, pp. 4680-4691, 2013.

[26] H. Amann, "Fixed point equations and nonlinear eigenvalue problems in ordered Banach spaces," SIAM Review, vol. 18, no. 4, pp. 620-709, 1976. 